\title{
Regulaciones y Guías para la Quimigación ${ }^{1}$
}

\author{
Haimanote K. Bayabil, Kati W. Migliaccio, Jonathan H. Crane, Teresa Olczyk, Qingren Wang, \\ Laura Vásquez, Carlos Balerdi²
}

\section{Introducción}

La "quimigación" es un proceso que usa el sistema de riego para transportar y llevar agroquímicos, generalmente fertilizantes y pesticidas, a una cosecha (Haman and Zazueta 2017). Los sistemas de riego de goteo y por aspersión son frecuentemente usados en la quimigación. Si el sistema de riego y la quimigación se diseñan bien pueden ayudar a los cultivadores a reducir el uso de químicos y los costos, porque se aplican usando la infraestructura de distribución existentes. Sin embargo, el sistema de riego debe ser equipado con accesorios de seguridad propiamente diseñados de fuentes de agua. Además, es importante para evitar la contaminación que el sistema de riego sea equipado con una bomba de inyección diseñada para injectar pesticidas o fertilizantes a un flujo apropiado basado en una unidad de área básica. La concentración del pesticida o fertilizante en el tanque de mezcla debe hacerse a flujos registrados porque la aplicación o concentraciones más altos pudieran dañar a las cosechas y como resultado en el lavado producido por debajo de la zona radical lo que pudiera contaminar cuerpos de agua potable. El propósito de este documento es proveer una breve reseña de regulaciones y alguna ayuda para la aplicación a flujos aprobados.

\section{Regulaciones para la Quimigación}

Dependiendo del tipo de quimíco a injectar hay requerimientos que tienen que usarse de acuerdo a la ley de Florida. Para cualquier químico hay que seguir las guías aprobadas. Los sistemas de quimigación tienen que tener un mecanismo antisifon para prevenir flujos de agua en reverso a la tubería principal. Los mecanismos antisifón son críticos para mitigar el riesgo de contaminación de agua potable. De acuerdo a reglas de Florida (Estatudos de Florida número 487.064 [https://www.myfloridahouse.gov/ Statutes/2019/0487.064/], 570.07, F.S. [https://www.flsenate. gov/laws/statutes/2020/570.07], inicialmente 5E-2.30), todo sistema de riego usado para aplicar pesticidas debe estar equipado con un mecanismo antisifón que asegure que el agua potable está protegida de una possible contaminación. Los requerimintos también cubren a las tuberias para mezclar y llenar equipos, a no ser que los sistemas incorporen un espacio físico entre la fuente de agua y el equipo de aplicación. El equipo antisifón debe tener una valvula automática en la bomba de inyeción que prevenga que los pesticidas injectados al sistema, de agua cuando el flujo de agua cesa. Por lo tanto es ilegal que una persona aplique pesticidas con un sistema de riego que no este equipado con el equipo antisifón requerido por esta regla.

1. This document is AE567, one of a series of the Department of Agricultural and Biological Engineering, UF/IFAS Extension. Original publication date November 2021. Visit the EDIS website at https://edis.ifas.ufl.edu for the currently supported version of this publication.

2. Haimanote K. Bayabil, profesor asistente, Departamento de Agricultura e Ingeniería Biologica, UF/IFAS Centro de Educación e Investigación Tropical; Kati W. Migliaccio, presidente y profesor, Departamento de Agricultura e Ingeniería Biologica; Jonathan H. Crane, profesor, Departmento de Ciencia Hortícolas, UF/IFAS Centro de Educación e Investigación Tropical; Teresa Olczyk, director, UF/IFAS Extension Condado de Miami-Dade; Qingren Wang, UF/IFAS agente de Extensión III para producción comercial de vegetales y certificación de licencias, UF/IFAS Extension Condado de Miami-Dade; Laura Vasquez, supervisor vecindarios y jardines en Florida, UF/IFAS Extension Condado de Miami-Dade; Carlos Balerdi, profesor emerito de cultivos frutales tropicales, UF/IFAS Extension Condado de Miami-Dade; UF/IFAS Extension, Gainesville, FL 32611.

The Institute of Food and Agricultural Sciences (IFAS) is an Equal Opportunity Institution authorized to provide research, educational information and other services

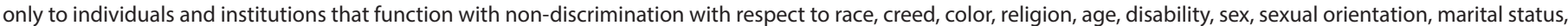

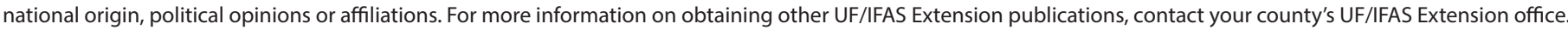
U.S. Department of Agriculture, UF/IFAS Extension Service, University of Florida, IFAS, Florida A \& M University Cooperative Extension Program, and Boards of County Commissioners Cooperating. Nick T. Place, dean for UF/IFAS Extension. 


\section{Ley para la Prevención del Retroceso de Flujo}

Los sistemas de quimigación deben cumplir con la ley del estado de Florida (Estatudos de Florida número 487.021 y 487.055) y la nota número 87-1 que explica la regulación de pesticidas bajo el reglamento establecido por la Agencia de Protección Ambiental (EPA por sus sigleas en ingles), que require que la prevención de flujo en reverso tiene que estar escrito en la etiqueta del químico. Las reglas generales para la prevención de flujo en retroceso en Florida se ofrecen en las publicaciones electronicas de UF/IFAS Extension (Haman and Zazueta 2017; Haman 2017). Además, ordenanzas locales deben consultarse porque ellas pueden ser más restrictivas. La injección de químicos por el lado de succión de una bomba centrífuga generalmente no se permite en Florida.

\section{Ventajas y Desventajas de la Quimigación}

La quimigación tiene ventajas y desventajas. Discutiremos brevemente algunas ventajas y deventajas de la quimigación.

\section{Ventajas}

- Como el químico se aplica con el agua de riego, el sistema de riego facilita una aplicación uniforme dentro del area de la zona de raíces.

- El sistema de micro riego debe ser automático y puede ser usado para regar la cosecha y cumplir con el riego y con la aplicación de agroquímicos. La inyección de pesticidas con el riego facilita la aplicación de químicos a bajo costo, opuesto a una aplicación separada con equipo para regar el suelo, que requiere trabajo y combustible para operar y distribuir los químicos.

- La quimigación de quimicos y fertilizantes evita el tráfico de equipos pesados por la finca y por lo tanto minimiza la compacción del suelo.

- Cuando el químico se inyecta al sistema de riego permanece en el sitema cerrado hasta que llega al área de aplicación. Por lo tanto la quimigación es un sistema conveniente para minimizar el contacto de los trabajadores con los químicos.

\section{Desventajas}

- Alto costo iniciales del sistema de inyección y de la infraestructura.
- Requiere más conocimiento y manejo del sistema de riego, la inyección de químicos, dilución de pesticidas y el envío, a través del riego a las áreas seleccionadas.

- Si el riego no se maneja bien para minimizar el acarreo superficial y, o la percolación profunda del agua al área de enraizamiento, los pesticidas y fertilizantes se pueden perder por lavado. Por lo tanto, la cantidad de químico que queda en la zona de las raíces puede ser menor que la recomendada, que puede contribuir a reducir la efectividad de los químicos.

- Hay riesgo que el equipo no funcione si el sistema no se atiende o se usa mal, que puede resultar en la contaminación del agua disponible.

- Ya que los pesticidas está duluidos y distribuidos por el sistema de riego, hay riesgo de aplicación excesiva de agua si la cosecha no requiere agua en ese momento.

\section{Consejos Prácticos para la Quimigación}

\section{Consejo 1: Asegúrese que el sistema de riego tiene válvulas de control y filtros}

Las válvulas de control son componentes que se colocan en la tubería para permitir el flujo de agua en solo una dirección. Esto previene que el flujo de retorno ocurra para protejer a la fuente del agua y a los químicos. Las válvulas de control se tienen que colocar entre la fuente de agua y el punto de injección del químico (Figura 1).

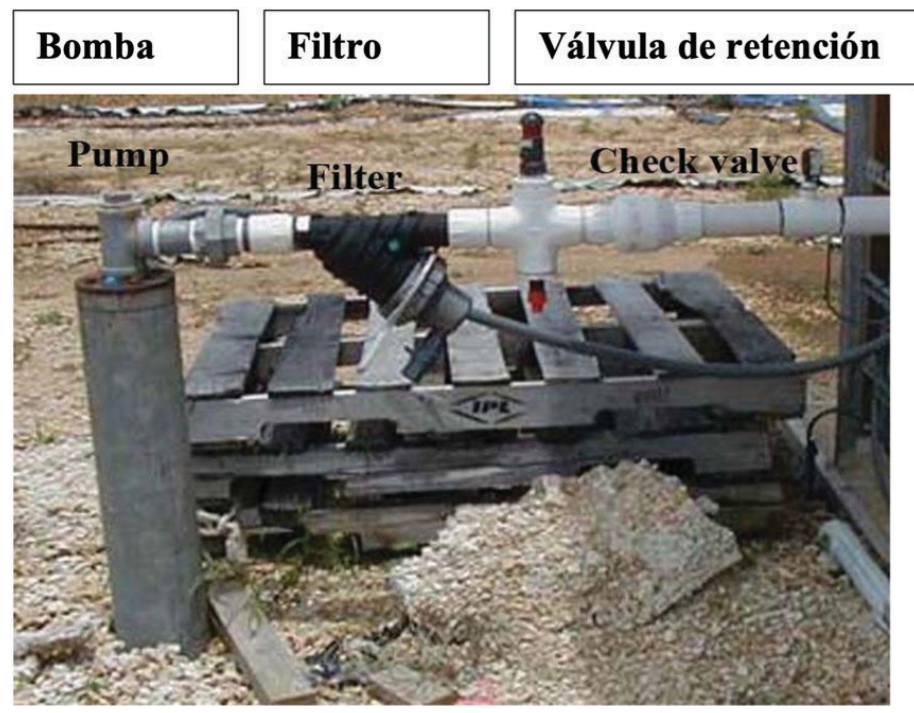

Figura 1. Fuente de agua, bomba, y conjunto de la válvula de retención.

Credits: Kati Miqliaccio, UF/IFAS

Debe colocarse un filtro en la tubería de riego. Los filtros se colocan directamente después de la fuente de agua para proteger al sistema de riego de tupiciones. Los filtros evitan la tupición de las tuberias lejos de los filtros y proveen 
acceso fácil para limpiar desechos. Los otros componentes requeridos por la ley de Florida incluyen drenajes de baja presión, eliminadores de vacio y válvulas de control de la fuente de químicos.

\section{Consejo 2: Calcular la cantidad de químico necesaria}

Durante la químigacion, el químico se injecta en la línea de riego. El saber la cantidad exacta requerida del químico es crítico (ver ejemplos en la Figura 2). El cálculo de la cantidad correcta requerida del químico para la quimigación se puede hacer como sigue.

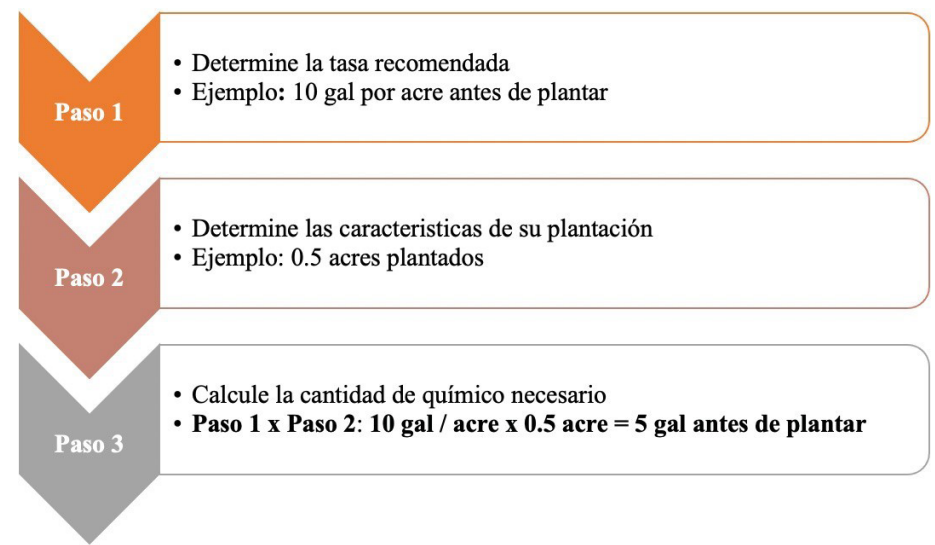

Figura 2. Ejemplo de un cálculo para determiner la cantidad de químico necesario para la aplicación en un área determinada. Credits: Haimanote Bayabil, UF/IFAS

Paso 1: Determinar el flujo correcto del químico para una aplicación específica. Debe ser expresada como cantidad por unidad de área (lb/acre o gal/acre).

Paso 2: Determinar el area o el numero de plantas usando quimigación. Recuerde, al calcular el área, incluya sólo el área sembrada con riego por goteo y plástico. Luego, calcule el área de siembra como longitud de surco multiplicado por el número de surcos.

Paso 3: Calcule la cantidad de químico necesitada basada en la información del primer paso y el segundo paso. Paso 1 multiplicado por el Paso 2.

\section{Consejo 3: Calibre la bomba de inyección}

Los inyectores de químicos son, en general de dos tipos: Inyector Venturi o con bomba calibrada (inyector de bomba de desplazamiento positivo).

Los inyectores de químicos deben calibrarse antes de usarlos y periódicamente durante su uso. La calibración de un inyector de químicos es un proceso para determinar cuanto químico se está inyectando en distintas concentraciones. El balance entre el químico inyectado y la cantidad de flujo de agua son muy importantes.

Si hay muy poca agua en la solución con el químico, pueden ocurrir los siguientes problemas:

- Distribución no uniforme del químico

- Alta volatibilidad (pérdida de químico)

- Aumento del químico en las tuberías

- Potencial de daño a cosecha debido a la concentración del químico

Si hay demasiada agua en la solución con el químico, los siguientes problemas pueden ocurrir:

- Dilución del químico por debajo de la concentración efectiva

- Pérdida del químico debido al lavado y a la contaminación de la fuente de agua

\section{Ejemplo: Calibración de una Bomba de Inyección}

La calibración de una bomba de inyección se puede hacer usando el siguente equipo: inyector, bolígrafo y papel, contenedor marcado con niveles volumetricos, y reloj:

1. Llene el contenedor a un determinado volumen.

2. Gradúe el inyector al flujo deseado (usualmente expresado en un dial como un número).

3. Decida el volumen de agua a remover con el inyector (asegúrese de que el volumen es apropiado para el contenedor).

4. Ponga la tubería del inyector dentro del contenedor.

5. Arranque el inyector y el reloj al mismo tiempo.

6. Pare el reloj cuando el volumen en el contenedor llega al nivel deseado.

7. Escribe en el papel el nivel del inyector, el volumen removido del contenedor, y el tiempo requerido.

8. Repita el proceso para diferentes niveles del reloj.

9. Calcule el flujo al cual el inyector opera (volumen/ tiempo) a cada nivel del dial.

El resultado de la calibración de un inyector de nutrients se muestra en la Tabla 1. 


\section{Consejo 4: Sea muy cuidadoso con las unidades}

Las unidades que se dan con cualquier producto, como $\mathrm{lb}$, kg, gal, etc., deben ser vistas cuidadosamente. La conversion incorrecta en unidades o la negligencia y el no usar unidades apropiadas puede resultar en errores en quimigación. Se proveen algunas conversiones de unidades en la Tabla 2.

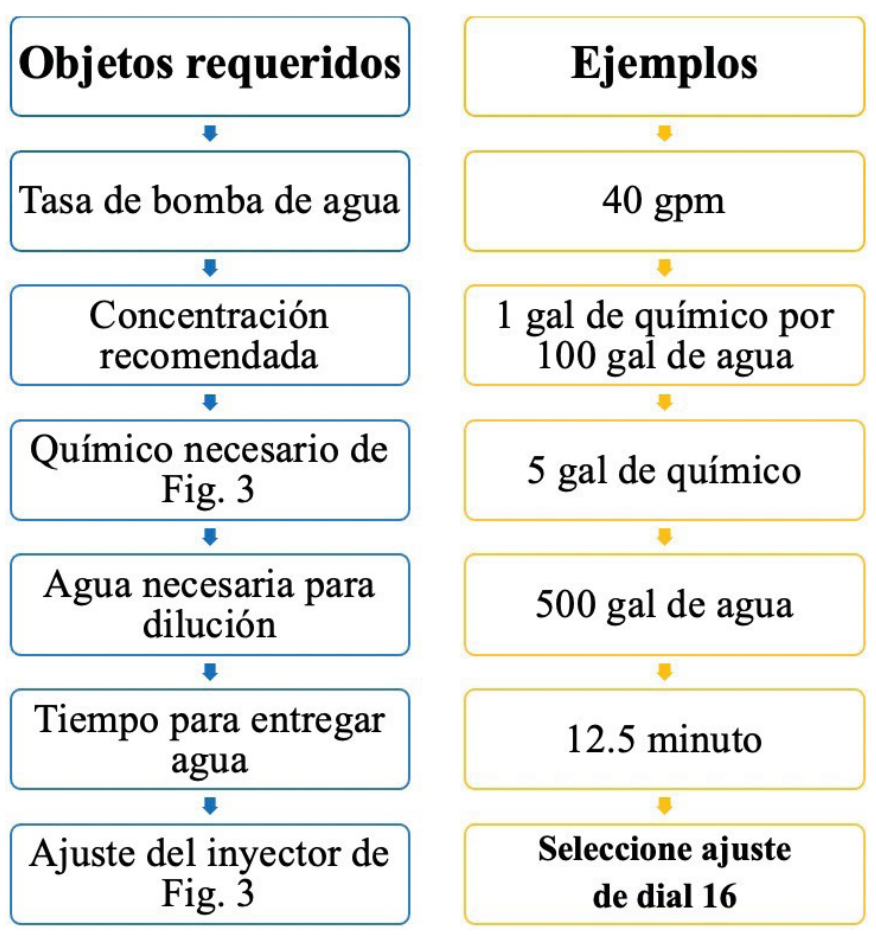

Figura 3. Ejemplo de pasos para determinar el ajuste apropiado de inyectores. Los pasos para el cálculo de la cantidad de producto químico necesario se muestran anterior en la Figura 2.

Credits: Haimanote Bayabil, UF/IFAS

\section{Consejo 5: Usar combinaciones apropiadas de químico y agua}

Para usar la combinación correcta químico y agua, el flujo de la bomba de riego y la dilución recomendada (o concentración) tienen que determinarse. Esta información debe estar disponible por la compañía o por su agente local de Extensión. Un ejemplo del cálculo para determinar el nivel del inyector del químico se muestra en la Figura 3. Además, recuerde que algunos pesticidas no se disuelven facilmente en agua. Si no está seguro de la solubilidad de un compuesto, hable con el distribuidor del químico o su agente local de Extensión. Si los pesticidas usados no son solubles, ellos pueden acumularse en el sistema resultando en tuberías a obstruidas.

\section{Consejo 6: Mantenga su sistema en buenas condiciones}

El continuo mantenimiento es necesario para la buena operación de un sistema de quimigación. Por lo tanto, los compenentes del riego hay que evaluarlo regularmente, y los problemas hay que arreglarlos cuando occuran. Al arreglar partes del sistema de riego, es importante que las partes sean de la misma especificación. Un buen método para evaluar el riego es arrancar el riego y caminar por el terreno si es apropiado. Mientras camina, busque, observe y escuche las fugas de liquido. La mayoría de las fugas del sistema de riego producirán un tipo de silvato y habrá un charco. Asegúrese de que las inpecciones de los componentes de riego se deben completar sin que el inyector de químicos esté apagado. Otra buena idea de mantenimiento es colocar metros de presión en las tuberías y usar estos metros para detectar problemas en el sistema.

\section{Precauciones Adicionales para Usar la Quimigación}

\section{Limpie el Sistema de Riego Después de Cada Quimig-} ación: Es extremadamente importante limpiar el sistema completo de quimigación, la bomba de inyección, los coladores, las mangueras, y las válvulas de control con agua limpia después de cada quimigación. Esto garantiza que no quedarán residuos de pesticidas en el sistema de riego. La limpieza del sistema de riego tiene que hacerse con el riego funcionando de manera que la solución con residuos es aplicada al campo.

Mantenga la Presión de Agua Óptima: Esto es importante para asegurar que los rociadores trabajen como deben y que la distribución de agua sea uniforme y que cubra el area indicada en las especificaciones. Extrema alta presión produce neblina y malgasta ambos, agua y químicos durante la quimigación.

\section{Haga Inspecciones Periodicamente del Sistema de}

Riego: La efectividad de la quimigación depende del funcionamiento apropiado del sistema de riego. Asegúrese de que el modelo de aspersión o de goteo son mantenidos apropiadamente, y repare las fugas y/o aspersores defectuosos. Asegúrese de que el modelo de aspersión del sistema de riego no sobrepase el área prevista del campo. Esto es muy importante en los sistemas y con los cañones. Evite la quimigación con aspersores durante vientos fuertes, que puedan aumentar el agua y los químicos perdidos por acarreo. El sistema de riego tiene que ser manejado para evitar acarreo y percolación profunda para minimizar la 
pérdida de agua y químicos fuera de área o por debajo del área de las raíces.

Siga Estrictamente la Etiqueta de Químicos: La quimigación de un químico que no está aprobado esta estrictamente prohibida porque esta práctica puede impactar negativamente la calidad del agua, las plantas no incluidas y a la fauna silvestre.

Siga la Etiqueta para Mezclar Químicos: Algunos pesticidas no son compatibles para mezclar. Por lo que las recomendaciones da la etiqueta deben ser estrictamente seguidas para evitar mezclar pesticidas erroneamente que no sean compatibles.

\section{Para Más Información}

Para obtener más información sobre la quimigación, pueden ser útiles publicaciones adicionales de EDIS. Se encuentran disponibles publicaciones más detalladas sobre quimigación y fertirrigación que incluyen, entre otras:

Clark, G. A., D. Z. Haman, and F. S. Zazueta. 2017. Injection of Chemicals into Irrigation Systems: Rates, Volumes and Injection Periods. BUL250. Gainesville: University of Florida Institute of Food and Agricultural Sciences. https://edis.ifas. ufl.edu/ae116

DeValerio, J., D. Nistler, R. Hochmuth, and E. Simonne. 2018. Fertigation for Vegetables: A Practical Guide for Small Fields. HS1206. Gainesville: University of Florida Institute of Food and Agricultural Sciences. https://edis.ifas.ufl.edu/ hs1206

Haman, D. Z. 2017. Causes and Prevention of Emitter Plugging in Microirrigation Systems. BUL258. Gainesville: University of Florida Institute of Food and Agricultural Sciences. https://edis.ifas.ufl.edu/ae032

Haman, D. Z., and F. S. Zazueta. 2017. Chemical Injection Methods for Irrigation. CIR864. Gainesville: University of Florida Institute of Food and Agricultural Sciences. https:// edis.ifas.ufl.edu/wi004

Hochmuth, G., R. Mylavarapu, and E. Hanlon. 2019. The Four Rs of Fertilizer Management. SL411. Gainesville: University of Florida Institute of Food and Agricultural Sciences. https://edis.ifas.ufl.edu/ss624
Liu, G., and G. K. England. 2018. How to Convert Liquid Fertilizer into Dry Fertilizer for Commercial Vegetable and Fruit Crop Production. HS1200. Gainesville: University of Florida Institute of Food and Agricultural Sciences. https:// edis.ifas.ufl.edu/hs1200

Liu, G., K. Morgan, Y. Li, L. Zotarelli, Q. Wang, and J. DeValerio. 2019. What is $4 R$ nutrient stewardship? HS1264. Gainesville: University of Florida Institute of Food and Agricultural Sciences. https://edis.ifas.ufl.edu/hs1264

Liu, G., E. H. Simonne, K. T. Morgan, G. J. Hochmuth, S. Agehara, and R. Mylavarapu. 2019. Chapter 2. Fertilizer Management for Vegetable Production in Florida. CV296. Gainesville: University of Florida Institute of Food and Agricultural Sciences. https://edis.ifas.ufl.edu/cv296

Zotarelli, L., M. D. Dukes, G. Liu, E. H. Simonne, and S. Agehara. 2019. Chapter 3. Principles and Practices of Irrigation Management for Vegetables. CV297. Gainesville: University of Florida Institute of Food and Agricultural Sciences. https://edis.ifas.ufl.edu/cv297

\section{Referencias}

Haman, D. Z. 2017. Causes and Prevention of Emitter Plugging in Microirrigation Systems. BUL258. Gainesville: University of Florida Institute of Food and Agricultural Sciences. https://edis.ifas.ufl.edu/ae032

Haman, D. Z., and F. S. Zazueta. 2017. Chemical Injection Methods for Irrigation. CIR864. Gainesville: University of Florida Institute of Food and Agricultural Sciences. https:// edis.ifas.ufl.edu/wi004 
Tabla 1. Ejemplos de la calibración de inyector de los resultados de bomba medidora.

\begin{tabular}{|c|c|c|c|c|}
\hline \multirow[t]{2}{*}{ Adjuste de Dial } & \multicolumn{2}{|c|}{ Calculado en el Campo } & \multirow[t]{2}{*}{ Tasa (gal/min) } & \multirow{2}{*}{$\begin{array}{c}\text { Tiempo (min) para } \\
\text { Entregar } \\
5 \text { gal }\end{array}$} \\
\hline & Volumen (gal) & Tiempo (min) & & \\
\hline 2 & 0.5 & 10.2 & 0.05 & 102 \\
\hline 4 & 0.5 & 6.5 & 0.08 & 65 \\
\hline 8 & 0.5 & 3.7 & 0.14 & 37 \\
\hline 16 & 0.5 & 1.3 & 0.38 & 13 \\
\hline
\end{tabular}

Tabla 2. Referencia rápida para conversions de unidades.

\begin{tabular}{|c|c|c|c|}
\hline \multirow[t]{5}{*}{ Longitud } & 1 pulgada $=2.54$ centimetros & \multirow[t]{5}{*}{ Área } & 1 ácre $=0.405$ hectares \\
\hline & 1 pie $=0.305$ metros & & 1 ácre $=43,560$ pies cuadrados \\
\hline & 1 pie $=30.48$ centimetros & & 1 ácre $=0.002$ millas cuadradas \\
\hline & 1 yarda $=0.914$ metros & & 1 ácre $=4046.86$ metro cuadrado \\
\hline & 1 milla = 1.609 kilómetros & & 1 ácre $=4,840$ yarda cuadrada \\
\hline \multirow[t]{4}{*}{ Volumen } & 1 medio litro $=0.473$ litros & \multirow[t]{4}{*}{ Masa } & 1 líbra = 0.454 kilogramos \\
\hline & 1 galón = 0.134 pie cúbico & & 1 tonelada $=907.185$ kilogramos \\
\hline & 1 onza líquida $=29.57$ millilitros & & 1 libra/ácre = 1.12 kilogramos $/$ hectáre \\
\hline & 1 pulgadacúbica = 16.39 centimetroscúbico & & 1 onza $=28.35$ gramas \\
\hline
\end{tabular}

